\title{
Epidemiologia da pitiose equina na Região Sul do Rio Grande do Sul ${ }^{1}$
}

\author{
Clairton Marcolongo-Pereira², Eliza S. Viégas Sallis ${ }^{3}$, Margarida B. Raffi ${ }^{3}$, Daniela I. \\ Brayer Pereira ${ }^{4}$, Fabiane L. Hinnah ${ }^{5}$, Ana Carolina B. Coelho ${ }^{5}$ e Ana Lucia Schild ${ }^{6 *}$
}

\begin{abstract}
Marcolongo-Pereira C., Sallis E.S.V., Raffi M.B., Pereira D.I.B., Hinnah F.L., Coelho A.C.B. \& Schild A.L. 2012. [Epidemiology of equine pythiosis in southern of Rio Grande do Sul State, Brazil.] Epidemiologia da pitiose equina na Região Sul do Rio Grande do Sul. Pesquisa Veterinária Brasileira 32(9):865-868. Laboratório Regional de Diagnóstico, Faculdade de Veterinária, Universidade Federal de Pelotas, Campus Universitário s/n, Pelotas, RS 96010-900, Brazil. E-mail: alschild@terra.com.br

A survey of cases of equine pythiosis received by the Laboratório Regional de Diagnóstico, Faculdade de Veterinária, Universidade Federal de Pelotas, from January 1979 to July 2011, was made to determine the epidemiological conditions in which the disease occurs in southern Rio Grande do Sul. Samples from 1888 horses were received; 435 samples were from the integumentary system, of which $63(14.5 \%)$ corresponded to pythiosis. The affected animals were of both sexes and their age ranged from 8 months to 22 years. Crioulo was the most prevalent breed. Most cases of pythiosis were sent to the laboratory between March and June. The evolution of the lesions due to pythiosis ranged from 2 weeks to 1 year. The municipalities with the greatest number of cases were Pelotas $(22 / 63)$, Santa Vitória do Palmar (15/63) and Rio Grande (8/63). With respect to the climatic data, in most cases the maximum temperature in the probable month of infection was above or close to $30^{\circ} \mathrm{C}$ during at least one day. The observation of cases in the colder seasons of the year could be due to the stagnant water temperature higher than the temperature of the air, which allows the development of infective structures of Pythium insidiosum.
\end{abstract}

INDEX TERMS: Pythium insidiosum, epidemiology, pythiosis, horses.

RESUMO.- Foi realizado um levantamento dos casos de pitiose equina recebidos no Laboratório Regional de Diagnóstico da Faculdade de Veterinária da Universidade Federal de Pelotas, no período de janeiro de 1979 a julho de 2011, com o objetivo de determinar as condições epidemiológicas em que a doença ocorre na região sul do Rio Grande do Sul. Nesse período foram recebidos 1888 materiais de equinos, dos quais, 435 eram provenientes do sistema tegumentar e $63(14,5 \%)$ corresponderam à pitiose. Os animais afetados

\footnotetext{
${ }^{1}$ Recebido em 11 de janeiro de 2012.

Aceito para publicação em 24 de maio de 2012

${ }^{2}$ Pós-Graduando em Medicina Veterinária, Faculdade de Veterinária, Universidade Federal de Pelotas (UFPel), Campus Universitário s/n, Pelotas, RS 96010-900, Brasil.

${ }^{3}$ Professoras do Departamento de Patologia Animal, FV-UFPel, Pelotas, RS.

${ }^{4}$ Professora do Departamento de Microbiologia, IB-UFPel, Pelotas, RS.

${ }^{5}$ Alunas do Curso de Medicina Veterinária, Universidade Federal de Pelotas (UFPel), Pelotas, RS. Bolsistas PIBIC-CNPq.

${ }^{6}$ Laboratório Regional de Diagnóstico, Faculdade de Veterinária, UFPel, Pelotas, RS. *Autor para correspondência: alschild@terra.com.br
}

eram de ambos os sexos com idades variando entre oito meses e 22 anos. A raça mais frequentemente afetada foi a Crioula. A maioria dos casos de pitiose foi encaminhada ao laboratório entre março e junho. A evolução das lesões de pitiose variou de duas semanas até um ano. Os municípios com maior número de casos de pitiose foram Pelotas (22/63) Santa Vitória do Palmar (15/63) e Rio Grande (8/63). Foi observado que na maioria dos casos, no mês provável de infecção a temperatura máxima foi superior ou próxima a $30^{\circ} \mathrm{C}$ em pelo menos um dia. A observação de casos em épocas mais frias do ano pode ser devido ao fato da temperatura de águas estagnadas ser mais elevada que a temperatura ambiental o que permite o desenvolvimento das estruturas infectantes de Pythium insidiosum.

TERMOS DE INDEXAÇÃO: Pythium insidiosum, epidemiologia, pitiose, equinos.

\section{INTRODUÇÃO}

As principais lesões de pele diagnosticadas em equinos no RS incluem sarcoide, carcinoma de células escamosas, 
pitiose e tecido de granulação (Souza et al. 2011). Dentre essas dermatopatias destaca-se a pitiose, uma enfermidade infecciosa de difícil tratamento causada pelo oomiceto aquático Pythium insidiosum. É descrita em regiões de clima tropical, subtropical e temperado, em países do continente Americano, Oceania (Mendoza et al. 1996) e África (Rivierre et al. 2005), afetando diversas espécies domésticas e selvagens e também humanos. No Brasil, a pitiose tem sido bem documentada e ocorre em equinos (Santos et al. 1987, Leal et al. 2001, Sallis et al. 2003), bovinos (Gabriel et al. 2008, Grecco et al. 2009), ovinos (Tabosa et al. 2004), caninos (Pereira et al. 2010) e humanos (Marques et al. 2006), porém a maioria dos casos corresponde a lesões cutâneas em equinos (Santurio et al. 2006).

0 acúmulo de água em banhados e lagoas, a presença de vegetação aquática e temperaturas entre 30 e $40^{\circ} \mathrm{C}$, são fatores essenciais que influenciam a ocorrência da pitiose (Miller \& Campbell 1982). Nestes ambientes P. insidiosum realiza seu ciclo de reprodução formando zoósporos infectantes, que ao serem liberados nas águas são atraídos por quimiotaxia para tecidos danificados, onde se fixam e emitem tubos germinativos dando início a enfermidade (Mendoza et al. 1996, Miller 1983). Comumente, observa-se que os animais afetados permanecem por longos períodos em contato com águas estagnadas (Chaffin et al. 1995).

Embora não exista um levantamento preciso da incidência e prevalência da enfermidade no Brasil, a mesma representa um problema à equinocultura, especialmente em regiões alagadas como o Pantanal Matogrossense (Mendoza et al. 1996). Na região sul do Rio Grande do Sul, cuja economia caracteriza-se pela produção integrada de agricultura-pecuária, com grandes extensões de áreas de arroz irrigado e uma população de equinos aproximada de 77.000 cabeças (IBGE 2009), a importância da pitiose equina é evidente, entretanto dados epidemiológicos são escassos.

O objetivo do presente estudo foi estudar os aspectos epidemiológicos da pitiose equina na área de influência do Laboratório Regional de Diagnóstico da Faculdade de Veterinária da Universidade Federal de Pelotas (LRD/UFPel).

\section{MATERIAL E MÉTODOS}

Foi realizado um estudo retrospectivo dos casos de pitiose equina ocorridos nos municípios da área de influência do Laboratório Regional de Diagnóstico (LRD) da Faculdade de Veterinária, Universidade Federal de Pelotas (UFPel), no período de 1979 a julho de 2011. 0 estudo foi baseado em consulta aos protocolos de necropsia e/ou biopsia arquivados no LRD. Foram coletadas informações referentes a dados epidemiológicos, sinais clínicos e localização das lesões. Em 47 casos o diagnóstico foi realizado pela cultura em corn meal agar (CMA) e/ou imuno-histoquímica pela técnica descrita por Brown et al. (1988) utilizando anticorpo policlonal anti-P. insidiosum. Nos demais materiais o diagnóstico foi realizado pelas lesões histológicas observadas na coloração com hematoxilina e eosina (HE) e impregnação pela prata (Grocott) levando-se em consideração a epidemiologia e a localização das lesões.

Com base na evolução da enfermidade e no período provável de incubação da doença em equinos, de aproximadamente um mês de acordo com o período de incubação observado em coe- lhos utilizados como modelo experimental (Pereira et al. 2008), estimou-se o momento de infecção do animal pelo agente.

Foi realizado um levantamento dos dados climáticos dos anos em que ocorreram os casos de pitiose nos Boletins Agroclimatológicos da Estação Agroclimatológica de Pelotas, Convênio Embrapa/UFPel/INMET obtendo-se o valor da temperatura máxima ocorrida no mês de provável início das lesões.

\section{RESULTADOS}

De janeiro de 1979 a julho de 2011 foram recebidos pelo LRD, 1888 materiais de equinos. Desses, 435 eram provenientes do sistema tegumentar, dentre os quais 63 corresponderam a pitiose, sendo 56 oriundos de biopsias e sete de necropsias. Em 14 casos, em que houve dúvida, o diagnóstico foi confirmado pela imuno-histoquímica. Os animais afetados eram de ambos os sexos com idades variando entre oito meses e 22 anos. A raça mais frequentemente afetada foi a Crioula.

Os casos de pitiose foram encaminhados ao laboratório em todos os meses do ano sendo cinco em janeiro, seis em fevereiro, 17 em março, cinco em abril, 15 em maio, sete em junho, dois em julho, dois em agosto e um caso em cada um dos meses seguintes até dezembro (Fig.1). A evolução das lesões de acordo com o apresentado nos protocolos de necropsia foi de três semanas (15 casos), um mês (oito casos), dois meses (seis casos), três meses (quatro casos), quatro e cinco meses (três casos cada) e sete, oito, nove e 12 meses (um caso cada). Em 20 casos não havia informação sobre o tempo de evolução das lesões nos protocolos de necropsia. Os municípios de procedência dos casos de pitiose equina diagnosticados no LRD entre 1979 e julho de 2011 são apresentados na Figura 2. No Quadro 1 são apresentados os dados de temperatura máxima no mês estimado da provável infecção dos equinos por Pythium insidiosum

Cinquenta e cinco equinos apresentavam uma única lesão, principalmente na porção distal dos membros. Três animais apresentaram lesões múltiplas nos membros torácicos e pélvicos e um equino manifestava lesão no lábio e membro torácico. Em quatro protocolos não foi informado a localização da lesão.

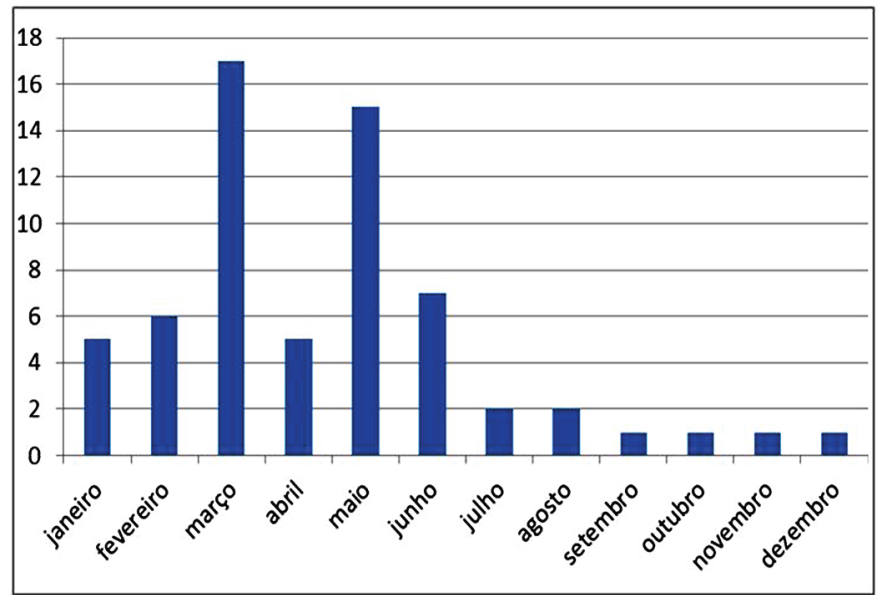

Fig,1. Número de casos de pitiose equina diagnosticados na área de influência do Laboratório Regional de Diagnóstico, Universidade Federal de Pelotas (UFPel), nos diferentes meses do ano. 


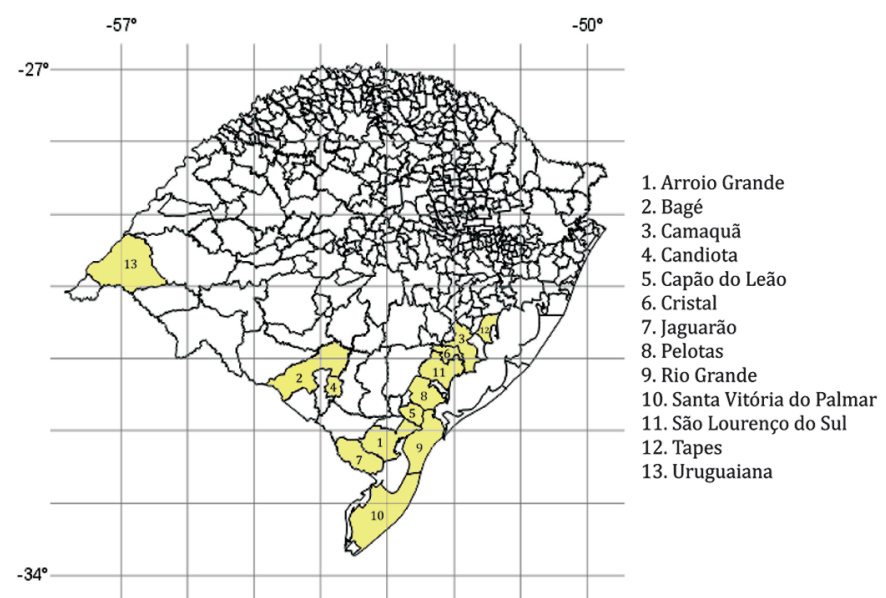

Fig.2. Municípios de procedência dos casos de pitiose equina recebidos no Laboratório Regional de Diagnóstico, UFPel, entre 1979 e julho de 2011.

Quadro 1. Temperatura máxima (TM) observada nos meses da provável infecção dos equinos por Pythium insidiosum considerando o período de incubação e a evolução da doença

\begin{tabular}{cccccc}
\hline Caso $n^{\mathbf{0}}$ & $\begin{array}{c}\text { Mês provável } \\
\text { da infecção }\end{array}$ & $\mathrm{TM}^{\circ} \mathrm{C}$ & ${\text { Caso } \mathrm{n}^{\mathbf{0}}}^{\text {Mês provável }}$ & $\begin{array}{c}\mathrm{T} \mathrm{M}^{\circ} \mathrm{C} \\
\text { da infecção }\end{array}$ & \\
\hline 01 & $10 / 1983$ & 32 & 23 & $03 / 2009$ & 31,6 \\
02 & $10 / 1983$ & 32 & 24 & $03 / 2009$ & 31,6 \\
03 & $03 / 1985$ & 33,2 & 25 & $02 / 2009$ & 33,6 \\
04 & $01 / 1988$ & 31,8 & 26 & $12 / 2008$ & 34,6 \\
05 & $01 / 1995$ & 35 & 27 & $12 / 2009$ & 33,6 \\
06 & $07 / 1994$ & 29,2 & 28 & $12 / 2009$ & 33,6 \\
07 & $02 / 1996$ & 34,6 & 29 & $01 / 2010$ & 32,9 \\
08 & $02 / 2001$ & 35,1 & 30 & $01 / 2010$ & 32,9 \\
09 & $03 / 2001$ & 32,6 & 31 & $12 / 2009$ & 33,6 \\
10 & $07 / 2002$ & 22,4 & 32 & $01 / 2010$ & 32,9 \\
11 & $02 / 2004$ & 33,4 & 33 & $01 / 2010$ & 32,9 \\
12 & $02 / 2006$ & 33,2 & 34 & $01 / 2010$ & 32,9 \\
13 & $12 / 2005$ & 35 & 35 & $01 / 2010$ & 32,9 \\
14 & $05 / 2006$ & 30,4 & 36 & $01 / 2010$ & 32,9 \\
15 & $02 / 2007$ & 36,2 & 37 & $01 / 2010$ & 32,9 \\
16 & $12 / 2006$ & 35 & 38 & $01 / 2010$ & 32,9 \\
17 & $01 / 2008$ & 33 & 39 & $01 / 2010$ & 32,9 \\
18 & $10 / 2007$ & 29,4 & 40 & $11 / 2010$ & 36 \\
19 & $04 / 2008$ & 32,4 & 41 & $03 / 2011$ & 34 \\
20 & $12 / 2008$ & 34,6 & 42 & $04 / 2011$ & 30,4 \\
21 & $02 / 2009$ & 33,6 & 43 & $12 / 2010$ & 32,4 \\
22 & $02 / 2009$ & 33,6 & & & \\
& & & & &
\end{tabular}

\section{DISCUSSÃO E CONCLUSÕES}

No presente estudo ficou demonstrado que a pitiose é uma das principais enfermidades que afetam a pele de equinos na área de influência do LRD, representando $14,5 \%$ dos casos diagnosticados em um período de 32 anos.

Em um estudo na região central do estado entre 1999 e 2009, a pitiose equina representou $8,3 \%$ dos casos de tumores cutâneos diagnosticados em equinos (Souza et al. 2011), e em um estudo de causas de morte nesta mesma espécie, realizado na mesma região entre 1968 e 2007, foi observado que de 335 casos de necropsias 3,58\% eram devidas à pitiose (Pierezan et al. 2009). No presente trabalho, o percentual de casos de pitiose entre 1999 e 2009 entre todos os casos de lesões de pele de equinos recebidas no LRD foi de $14,34 \%$. Isto demonstra a importância da pitiose equina na região sul do estado, provavelmente devido às características geográficas, com campos planos e sujeitos a alagamentos frequentes, propícios ao desenvolvimento do agente. Essas condições foram encontradas em todos os municípios onde a pitiose foi diagnosticada, particularmente naqueles de maior casuística como Pelotas (22/63), Santa Vitória do Palmar (15/63) e Rio Grande (8/63). Outro fato que reforça a importância da enfermidade na região é que um surto foi diagnosticado em bovinos, e de acordo com os autores, a doença pode ser mais frequente do que se supõe nesta espécie animal (Grecco et al. 2009), e quatro casos foram observados em caninos que tinham contato permanente ou esporádico com o campo (Pereira et al. 2010, Ladeira et al. 2011).

Animais infectados por Pythium insidiosum frequentemente desenvolvem lesões em locais que permanecem em contato, por longos períodos, com águas estagnadas (Chaffin et al. 1995). Na região estudada é comum observar-se equinos pastando dentro de lagos, várzeas e açudes. Neste estudo, as lesões de pitiose ocorreram predominantemente nos membros $(65,2 \%)$ e abdômen $(10,6 \%)$. Observou-se que a maioria dos equinos apresentava uma única lesão, o que é comum em outros relatos da doença (Miller \& Campbell 1982, Chaffin et al. 1995).

Apesar de que o maior número de casos chegou ao laboratório entre março e junho, deve-se levar em consideração o tempo de evolução das lesões que pode ser muito variável na enfermidade dos equinos (Miller \& Campbell 1982, Santos et al. 1987). Com relação ao período de incubação, não existem dados definitivos nesta espécie. A reprodução da enfermidade em modelo experimental utilizando coelhos demonstrou que a lesão se desenvolve em torno de 25 dias após a inoculação de zoósporos por via subcutânea (Pereira et al. 2008). Em bovinos ficou demonstrado que são necessários pelo menos 15 dias desde a entrada dos animais em uma área contaminada com o agente até o aparecimento das lesões (Grecco et al. 2009).

No presente trabalho, a evolução das lesões de pitiose, mencionada em 43 protocolos do LRD, desde a observação pelo proprietário ou veterinário até a remessa de biopsia ao laboratório variou de três semanas até um ano. Em 15 casos o tempo de evolução foi de três semanas, seguido de oito casos com evolução de um mês e seis com evolução de dois meses. Apesar de que muitas vezes lesões pequenas podem passar despercebidas, principalmente quando se trata de animais que permanecem soltos no campo que não são observados diariamente, aparentemente existe uma tendência de que a evolução da enfermidade possa variar de 3-8 semanas, embora períodos maiores possam ocorrer. Deve ser levado em consideração que o tempo de evolução das lesões depende também da capacidade de resposta imunológica dos animais. Tem sido sugerido que alguns animais seriam mais resistentes e outros teriam maior predisposição individual à infecção (Santos et al. 2011).

Com relação aos dados climáticos, foi observado que na maioria dos casos, no mês provável de infecção considerando o tempo de incubação de aproximadamente 25-30 dias e a evolução das lesões relatada, a temperatura máxima foi superior ou próxima a $30^{\circ} \mathrm{C}$ em pelo menos um dia. Esta temperatura ambiente é, aparentemente, suficiente para 
que o agente produza zoósporos infectantes que iniciam a doença após penetrarem na pele do animal (Miller 1983, Mendoza et al. 1996). Por outro lado, deve ser considerado que $P$. insidiosum é um organismo aquático e as formas infectantes (zoósporos) se desenvolvem dentro de águas paradas como açudes, banhados, canais de irrigação e campos alagados, no entanto a temperatura ideal dessas águas para o desenvolvimento da forma infectante do agente é desconhecida.

Em águas estagnadas a temperatura é variável de acordo com a profundidade do solo, com a irradiação solar, com a nebulosidade, com a temperatura ambiente, com a hora do dia e com a presença de ventos (Angelocci \& Villa Nova 1995). Tem sido demonstrado que a temperatura em águas paradas é superior a do ar, tanto em dias de alta irradiação solar como em dias nublados ou chuvosos (Angelocci \& Villa Nova 1995). Além disso, a água tem capacidade de reter calor contribuindo para que as flutuações térmicas sejam bem mais atenuadas do que as flutuações térmicas do ar (Angelocci \& Villa Nova 1995). Isso sugere que mesmo que a temperatura do ambiente seja inferior a $30^{\circ} \mathrm{C}$ ou que tenha variações durante as várias horas do dia, as águas onde $P$. insidiosum está presente podem ter a temperatura ideal para a zoosporogênese. Este fato poderia explicar o aparecimento de lesões de pitiose nos meses mais frios do ano, como provavelmente ocorreu em cinco casos descritos neste trabalho.

Com base nos resultados obtidos foi possível concluir que a infecção por $P$. insidiosum na região estudada, apesar de mais frequente nos meses mais quentes do ano, pode ocorrer em qualquer estação do ano, dependendo da temperatura da água onde o agente está presente. É importante salientar, também, que a doença pode afetar equinos de qualquer raça e idade e a observação frequente dos animais criados em áreas alagadas, adequadas ao desenvolvimento do agente, pode minimizar prejuízos uma vez que lesões iniciais podem ser tratadas cirurgicamente ou com imunoterápicos que podem curar até $70 \%$ dos equinos tratados (Mendoza et al. 1992, Mendoza et al. 2003).

\section{REFERÊNCIAS}

Angelocci L.R. \& Vila Nova N.A. 1995. Variações da temperatura da água de um pequeno lago artificial ao longo de um ano em Piracicaba, SP. Sci. Agric. 52(3):431-438.

Brown C.C., McClure J.J., Triche P. \& Crowder C. 1988. Use of immunohistochemical methods for diagnosis of equine pythiosis. Am. J. Vet. Res. 49:1866-1868.

Chaffin M.K., Schumacher J. \& Mcmullan W.C. 1995. Cutaneous pythiosis in the horse. Vet. Clin. North Am., Equine Pract. 11(1):91-103.

Gabriel A.L., Kommers G.D., Trost M.E., Barros C.S.L., Pereira D.B., Schwendler S.E. \& Santurio J.M. 2008. Surto de pitiose cutânea em bovinos. Pesq. Vet. Bras. 28(12):583-587.
Grecco F.B., Schild A.L., Quevedo P., Assis-Brasil N.D., Kommers G.D., Marcolongo-Pereira C. \& Soares M.P. 2009. Pitiose cutânea em bovinos na região Sul do Rio Grande do Sul. Pesq. Vet. Bras. 29(11):938-942.

IBGE 2009. Instituto Brasileiro de Geografia e Estatística. Disponível em <http://www.ibge.gov.br/cidadesat/topwindow.htm> Acesso em 27 jul. 2009.

Ladeira S.R.L., Ruas J.L., Soares M.P. \& Schild A.L. 2011. Boletim do Laboratório Regional de Diagnostico no.31. Editora e Gráfica Universitária, UFPel, Pelotas. 54p.

Leal A.B.M., Leal A.T., Santurio J.M., Kommers G.D. \& Catto J.B. 2001. Pitiose eqüina no pantanal brasileiro: Aspectos clínico-patológico de casos típicos e atípicos. Pesq. Vet. Bras. 21(4):151-156.

Marques S.A., Bagagli E., Bosco S.M.G., Camargo R.M.P. \& Marques M.E.A. 2006. Pythium insidiosum: relato do primeiro caso de infecção humana no Brasil. Anais Bras. Dermatol. 5:483-485.

Mendoza L., Villalobos J. \& Calleja C.E. 1992. Evaluation of two vaccines for the treatment of pythiosis insidiosi in horses. Mycopathologia 119:8995.

Mendoza L., Ajello L. \& McGinnis M.R. 1996. Infections caused by the oomycetous pathogen Pythium insidiosum. J. Med. Mycol. 6:151-164.

Mendoza L., Mandy W. \& Glass R. 2003. An improved Pythium insidiosum-vaccine formulation with enhanced immunotherapeutic properties in horses and dogs with pythiosis. Vaccine 21:2797-2804

Miller R.I. \& Campbell R.S.F. 1982. Clinical observations on equine phycomycosis. Aust. Vet. J. 58:221-226.

Miller R.I. 1983. Investigations into the biology of three 'phycomycotic' agents pathogenic for horses in Australia. Mycopathologia 81:23-28.

Pereira D.I.B., Santurio J.M., Alves S.H., Azevedo M.I., Silveira F., Costa F.F., Sallis E.S.V., Pötter L. \& Ferreiro L. 2008. Comparison between the immunotherapic Pitium Vac and the antifungal drug caspofungin as agents to treat experimental pythiosis in rabbits. Mycologie Médicale 18:129133.

Pereira D.I.B., Schild A.L., Motta M.A., Fighera R.A., Sallis E.S.V. \& Marcolongo-Pereira C. 2010. Cutaneous and gastrointestinal pythiosis in a dog in Brazil. Vet. Res. Commun. 34:301-306.

Pierezan F., Rissi D.R., Rech R.R., Fighera R.A., Brum J.S. \& Barros C.S.L. 2009. Achados de necropsia relacionados com a morte de 335 equinos: 1968-2007. Pesq. Vet. Bras. 29(3):275-280.

Rivierre C., Laprie C., Guiard-Marigny O., Bergeaud P., Berthelemy M. \& Guillot J. 2005. Pythiosis in Africa. Emerg. Infect. Dis.11(3):479-481.

Sallis E.S.V., Pereira D.I.B. \& Raffi M.B. 2003. Pitiose cutânea em eqüinos: 14 casos. Ciência Rural 33(5):899-903.

Santos M.N., Metzdorf L.L., Braga M.M. \& Wolle C.A. 1987. Pitiose cutânea em eqüinos no Rio Grande do Sul. Pesq. Vet. Bras. 7:57-61.

Santos C.E.P., Marques L.C., Zanette R.A., Jesus F.P.K. \& Santurio J.M. 2011. Does immunotherapy protect equines from reinfection by the Oomycete Pyhtium insidiosum? Clin. Vaccine Immunol. 18(8):1397-1399.

Santurio J.M., Alves S.A., Pereira D.B. \& Argenta J.S. 2006. Pitiose: uma micose emergente. Acta Scient. Vet. 34(1):1-14.

Souza T.M., Brum J.S., Fighera R.A., Brass K.E. \& Barros C.S.L. 2011. Prevalência dos tumores cutâneos de equinos diagnosticados no Laboratório de Patologia Veterinária da Universidade Federal de Santa. Pesq. Vet. Bras. 31(5):379-382. 2011.

Tabosa I.M., Medeiros V.T., Dantas A.F.M., Azevedo E.O. \& Maia J.C. 2004. Outbreaks of pythiosis in two flocks of sheep in Northeastern Brazil. Vet. Pathol. 41(4):412-415. 